\title{
Compound heterozygous $C A P N 3$ variants identified in a family with limb-girdle muscular dystrophy recessive 1
}

\author{
CHENG ZHANG ${ }^{1,2}$, XUEPING ZHENG ${ }^{3}$, DEGUO LU $^{2}$, LULU XU $^{3}$, FENGYUAN CHE $^{2 *}$ and SHIGUO LIU ${ }^{1 *}$ \\ ${ }^{1}$ Medical Genetic Department, The Affiliated Hospital of Qingdao University, Qingdao, Shandong 266003; \\ ${ }^{2}$ Department of Neurology, The Eleventh Clinical Medical College of Qingdao University, \\ Linyi People's Hospital, Linyi, Shandong 276000; ${ }^{3}$ Department of Geriatric Medicine, \\ The Affiliated Hospital of Qingdao University, Qingdao, Shandong 266003, P.R. China
}

Received October 5, 2020; Accepted April 12, 2021

DOI: $10.3892 / \mathrm{mmr} .2021 .12119$

\begin{abstract}
Limb-girdle muscular dystrophy recessive 1 (LGMDR1), a rare subtype of muscular dystrophy, is characterized by progressive muscle weakness and degeneration with a predominant presentation on the shoulder, pelvic and proximal limb muscles. Variants in calcium-activated neutral proteinase 3 (CAPN3), which encodes an enzyme, calpain 3 , are considered the major cause of LGMDR1. The present study was conducted to identify the variants responsible for clinical symptoms in a Chinese patient with limb-girdle muscular dystrophies (LGMDs) and explore its genotypephenotype associations. A series of clinical examinations were conducted, including blood tests and magnetic resonance imaging scans of the lower legs, electromyography and muscle biopsy on the proband diagnosed with muscular dystrophies. Genomic DNA was extracted from the peripheral blood of a three-person family with LGMDs and pathogenic variants
\end{abstract}

Correspondence to: Dr Fengyuan Che, Department of Neurology, The Eleventh Clinical Medical College of Qingdao University, Linyi People's Hospital, 27 East Section of Jiefang Road, Lanshan, Linyi, Shandong 276000, P.R. China

E-mail: che1971@126.com

Professor Shiguo Liu, Medical Genetic Department, The Affiliated Hospital of Qingdao University, 16 Jiangsu Road, Shinan, Qingdao, Shandong 266003, P.R. China

E-mail: shiguo2006liu@163.com

*Contributed equally

Abbreviations: CAPN3, calcium-activated neutral proteinase 3; CK, creatine kinase; LGMD, limb-girdle muscular dystrophies; LGMD2A, limb-girdle muscular dystrophy type 2A; LGMDR1, limb-girdle muscular dystrophy recessive 1 ; PEF, penta-EF-hand; WT, wild-type; MRI, magnetic resonance imaging; NGS, nextgeneration sequencing; WES, whole-exome sequencing

Key words: variant, calcium-activated neutral proteinase 3, caplain-3, limb-girdle muscular dystrophy recessive 1 , limb-girdle muscular dystrophy type $2 \mathrm{~A}$ detected by whole-exome sequencing (WES) were verified by Sanger sequencing. The WES of this patient revealed compound heterozygous variants in CAPN3, c.2120A>G/p. (Asp707Gly) in exon 20 and c.2201_2202delAT/p.(Tyr734*) in exon 21, which were inherited from his parents and absent from 200 control individuals of similar ethnic origin, indicating that these variants are the pathogenic triggers of the LGMDR1 phenotype. Notably, these CAPN3 sequence variants were related to LGMDR1 pathogenesis in this three-person family. The newly discovered c.2201_2202delAT/p.(Tyr734*) expands the current $C A P N 3$ variant spectrum, improving the understanding of the conditions required to develop molecular diagnostic tools and for genetic counseling, particularly for families with a history of autosomal recessive LGMDs.

\section{Introduction}

Calpainopathy, or limb-girdle muscular dystrophy recessive 1 [LGMDR1; Online Mendelian Inheritance in Man (OMIM):253600], previously known as limb-girdle muscular dystrophy type 2A (LGMD2A), has an incidence of 1 in 100,000 and is the commonest type of LGMD in Europe, accounting for approximately $30 \%$ of muscular dystrophies. However, LGMDs are relatively rare in China (1-3). The clinical manifestations of LGMDR1 are similar to those of other types of LGMDs, including progressive weakness and muscle atrophy in the shoulder, pelvic girdle muscles and proximal limb muscles. Notably, lower limb and body muscles are most affected. Some patients present with an abnormal gait or difficulty climbing stairs and lifting weights, accompanied by aggravated locomotor abilities (4-6). The phenotypic manifestations of LGMDR1 are highly heterogeneous and can be inconsistent even within the same family. Symptoms and physical signs may appear at any age and generally worsen over time, although they remain mild in some cases $(7,8)$. As the pathophysiological mechanism underlying LGMDR1 is currently unknown, no effective treatment is available for this disease.

Variants in calcium-activated neutral proteinase 3 (CAPN3; OMIM:114240) can cause LGMDR1 (autosomal recessive) and limb-girdle muscular dystrophy dominant 4 (LGMDD4; LGMD1I; OMIM:618129; autosomal dominant), the clinical 
manifestations of which are similar except for its morbidity, inheritance patterns and severity of symptoms (9). LGMDR1 is the first disease for which the genetic cause has been conclusively attributed to defective $C A P N 3$ via specific molecular biological effects (10). To date, more than 500 different $C A P N 3$ variants have been reported in the Leiden muscular dystrophy database (https://databases.lovd.nl/shared/genes/CAPN3) (11). The commonest type are missense variants $(\sim 60 \%)$, which are mostly compound heterozygous variants (12-14). The three different autosomal recessive subtypes associated with CAPN3 variants can be diagnosed based on the distribution of muscle weakness and age at the time of onset: Shoulder- and limb-girdle psoas muscular dystrophy (also known as LeidenMobius LGMD), shoulder and bone LGMD (also known as Erb LGMD) and hyper-creatine kinase-emia, which is typically observed in children or young adults (5). In most cases, the affected individuals do not present muscular symptoms but exhibit elevated serum creatine kinase (CK) levels $(5,15)$.

CAPN3, localized on chromosome 15q15.1 (16-18), spans more than $138 \mathrm{~kb}$ of genomic DNA with 24 exons and encodes a 94-kDa protease enzyme, calpain 3, consisting of 821 amino acids (NM_000070.2) (19). Calpain 3 protein, a calcium-dependent non-lysosomal neutral cysteine protease widely found in the sarcomere structure, serves a role in muscle regeneration, sarcolemmal repair, muscle assembly and remodeling, as well as cytoskeleton regulation and calcium homeostasis $(20,21)$. The main structure of calpain 3 includes four functional domains, a cysteine protease (II) and penta-EF-hand (PEF) (IV) domains, that respectively code for cysteine protease and calcium-binding domains involved in calcium-dependent enzyme activation $(22,23)$.

Due to the high clinical and genetic heterogeneity of LGMDs, it is challenging to distinguish different subtypes only on the basis of clinical symptoms and physical signs (24-26). As such, a large number of patients may be misdiagnosed in the early stage. Thus, genetic testing is strongly recommended to offer a reliable and conclusive differential diagnosis.

The present study described a 23-year-old man who presented with consistently elevated serum CK as his only symptom and was initially diagnosed with polymyositis. A strategy was devised to couple next-generation sequencing (NGS) with Sanger sequencing validation to identify and validate the disease-causing variants in this proband. The results provided insight for further research on the pathogenesis of LGMDs and to accelerate prenatal diagnosis development.

\section{Materials and methods}

Subjects. The proband (II:2), a 23-year-old male born to healthy, non-consanguineous parents (Fig. 1), was admitted to the Affiliated Hospital of Qingdao University due to consistently elevated, although fluctuating, CK levels. Based on laboratory examinations, the proband was initially diagnosed with polymyositis by the Immune Department of the Affiliated Hospital of Qingdao University (Shandong, China). However, a successive series of physical examinations and auxiliary tests, including serum CK measurement, electromyography, magnetic resonance imaging (MRI) and muscle biopsy, were performed to complement and validate the presumptive diagnosis. Control samples were randomly collected from patients

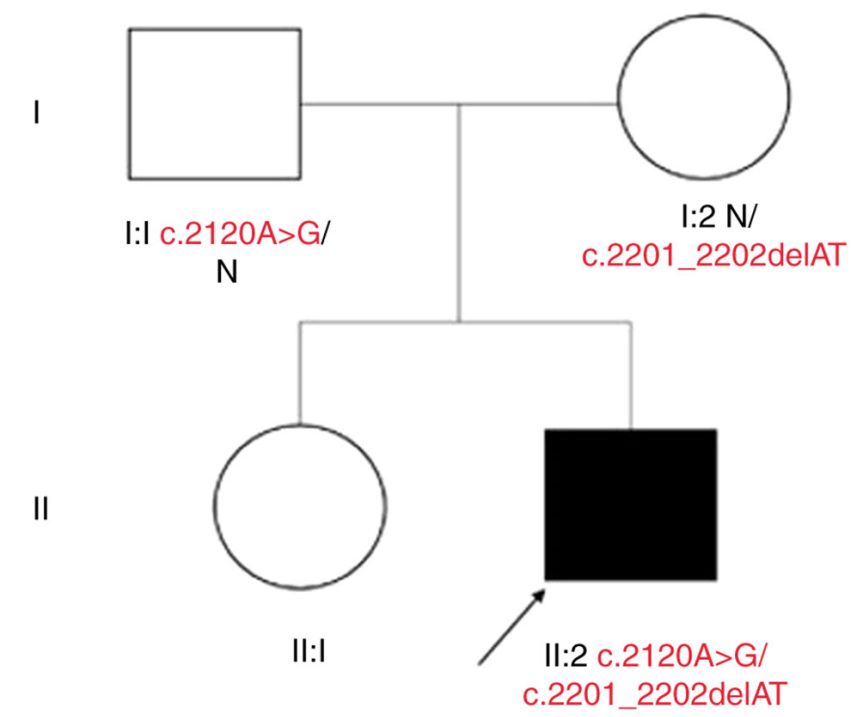

Figure 1. Family pedigree of the limb-girdle muscular dystrophy recessive 1 family.

who presented to The Affiliated Hospital of Qingdao University since August 2020. The control group comprised 200 healthy individuals (age, 18-65 years) from the ethnic Han Chinese population in Shandong Province. The male-to-female ratio was 1:1. The proband and parents as well as individuals in the control group signed informed consent forms prior to venous blood collection. The present study was approved by the ethics committee of the Affiliated Hospital of Qingdao University (approval no. qdfy20203789) and conformed to the guidelines set forth by the Declaration of Helsinki.

Genomic DNA preparation. From a three-person family, genomic DNA (gDNA) was extracted from $200 \mu 1$ of peripheral blood samples in the presence of ethylenediaminetetraacetic acid as an anticoagulant with a DNA Extraction kit (Qiagen $\mathrm{GmbH}$ ) according to the manufacturer's instructions. The optical density value of the gDNA was determined with a Sim-100 ultramicro spectrophotometer (Thermo Fisher Scientific, Inc.). gDNA samples were prepared as Illumina sequencing libraries. Targeted sequencing was performed on the proband samples (II:2). The pathogenic variant was validated by Sanger sequencing in both proband and parental samples.

Whole-exome sequencing (WES) for variant screening. WES technology was used to screen the mutated sites in CAPN3 (NM_000070.2, NP_000061.1). WES was performed according to the human exome capture protocol from Illumina's TruSeq Exome Enrichment Guide (SureSelectXT Target Enrichment System for Illumina Paired-End Sequencing Library, Agilent Technologies, Inc.). The exome enrichment probe sets were constructed with the Agilent Human All Exon $50 \mathrm{Mb}$ Exome Enrichment kit and sequenced on a HiSeq 4000 NGS platform (Illumina, Inc.). The captured gDNA library was sequenced on the Illumina HiSeq 4000 platform and $200(2 \times 100)$ bp were generated from the final library fragment using V2 Reagent 1.8 software (Illumina, Inc.; data after June 22, 2011). The average depth of the target area was 257.15 
and the target bases with coverage of at least $50 \mathrm{x}$ were $75.81 \%$, $20 \mathrm{x}$ were $82.23 \%$, 10x were $89.04 \%$, $4 \mathrm{x}$ were $93.56 \%$ and $1 \mathrm{x}$ were $96.09 \%$, respectively. Following sequencing, low-quality variants were filtered out to obtain clean reads. Paired-end sequence reads were mapped to the human reference genome hg19 with Burrows-Wheeler Aligner (27). Genome Analysis Toolkit software (https://gatk.broadinstitute.org/hc/en-us) (28) was used to identify single-nucleotide polymorphisms and insertions or deletions. All identified variants were submitted to ANNOVAR (version 2020Oct07) (29) for functional annotation and genetic filtering. All information presented in the present study was directly extracted from the reference data set or calculated in batches of all variants. Common variants were excluded by comparison with $>1,000$ exomes sequenced in our laboratory for unrelated conditions and subsequently filtered with the dbSNP v137 (ncbi.nlm.nih.gov/snp/), 1000 Genomes Project (ncbi.nlm.nih.gov/variation/tools/1000genomes/), National Heart, Lung and Blood Institute, Exome Sequencing Project (evs.gs.washington.edu/EVS/) and ExAC databases (exac.broadinstitute.org). Variant filtration was based on the following criteria: i) Excluding untranslated region 3 and 5 variants, non-coding RNA intron variants and intron and synonymous variants; ii) excluding minor allele frequency $>0.1$ variants (30); and iii) $\geq 50 \%$ of the harmful variants in the bioinformatics software [PolyPhen-2 (genetics.bwh.harvard. edu/pph2/), SIFT (sift.jcvi.org/www/SIFT_BLink_submit. html) and Mutation Taster (mutationtaster.org/ChrPos.html)] were retained.

Sanger sequencing validation. The variations detected by WES were validated by Sanger sequencing. Healthy controls (200) from the Han Chinese ethnic population in Shandong Province were randomly selected for validation. The male-to-female ratio was 1:1. The two pairs of primers covering the variants were: Forward, 5'-ATCCTGCCCAAGCAAAAGTG-3'; reverse, 3'-GCCGGACTGGTCTGTGTCAT-5' for c. $2120 \mathrm{~A}>\mathrm{G}$ in exon 20 (primer pair one) and forward, 5'-GGTAGGACAGCCCGGAGTCT-3'; reverse: 5'-TTGGG CCTGCCTTCTATTTTC-3' for c.2201_2202delAT in exon 21 (primer pair two). Identical amplification conditions for were used for both primer pairs in a total volume of $25 \mu \mathrm{l}$ containing $250 \mathrm{nM}$ dNTPs, $100 \mathrm{ng}$ of template DNA, $0.5 \mathrm{mM}$ of each primer and 1.25 units AmpliTaq Gold DNA polymerase in $1 \mathrm{X}$ reaction buffer $(10 \mathrm{mM}$ Tris $\mathrm{HCl}, \mathrm{pH} 8.3,50 \mathrm{mM} \mathrm{KCl}, 2.5 \mathrm{mM}$ $\mathrm{MgCl}_{2}$ ). PCR was performed as follows: initial denaturation at $94^{\circ} \mathrm{C}$ for $5 \mathrm{~min}$, followed by 35 cycles comprising denaturation at $94^{\circ} \mathrm{C}$ for $30 \mathrm{sec}$, annealing at $55^{\circ} \mathrm{C}$ for primer pair one and $58^{\circ} \mathrm{C}$ for primer pair two for $60 \mathrm{sec}$ and extension at $72^{\circ} \mathrm{C}$ for $30 \mathrm{sec}$; after which a final extension was conducted at $72^{\circ} \mathrm{C}$ for $10 \mathrm{~min}$. Amplified PCR products were purified and sequenced using appropriate PCR primers and a BigDye Terminator Cycle Sequencing kit (Applied Biosystems; Thermo Fisher Scientific, Inc.) and evaluated on an ABI 3730XL automated sequencer (Applied Biosystems; Thermo Fisher Scientific, Inc.) for variant analysis.

Bioinformatics analysis. Functional impacts of the variants were predicted in silico using the following software. Multiple sequence alignment was conducted to analyze gene sequence conservation. The CAPN3 amino acid sequences from multiple species were obtained from the UniProt website (http://www.uniprot.org). PolyPhen-2 (http://genetics. bwh.harvard.edu/pph2/) and SIFT (http://sift.jcvi.org/www/ SIFT_BLink_submit.html) software were used to analyze and predict the function of the identified variants.

The structure of a CAPN3 orthologue (PDB code 4OKH) was used to predict the structural role of CAPN3 residues (31). Using the SWISS-MODEL program (31), comparative modeling and energy minimization methods were used to model the three-dimensional structure of CAPN3 (32). The orthologous residues were determined by alignment with the amino acid sequence of ClustalW-2. PyMOL-1 (pymol. org/2/ V 2.4.1) was used to simulate and visualize the threedimensional protein structure of the wild-type (WT) and mutant proteins.

\section{Results}

Clinical features. The proband was a sporadic case with no related symptoms manifesting in the parents. The proband was admitted to the Affiliated Hospital of Qingdao University for consistently elevated, although fluctuating, CK levels. The clinical course was as follows: The proband was found to have elevated CK $(3,000 \mu \mathrm{g} / \mathrm{l}$; physiological range 38-174 $\mu \mathrm{g} / \mathrm{l})$ when he visited the hospital for a skin rash in 2015, without any muscle aches. Then, seven days later, the patient was admitted to the Department of Immunology of the hospital (CK: $2,185 \mu \mathrm{g} / \mathrm{l}$ ) and was initially diagnosed as possible polymyositis; thus, he was administered hormone combined with immunosuppressive therapy. No evident therapeutic effect was observed and his CK levels widely fluctuated during treatment. Until 2020, the patient presented no abnormalities, a normal walking posture and regular squatting and stair climbing abilities and could lift heavy objects. As the elevated CK levels did not affect his everyday life, this condition remained undiagnosed and untreated. On June 1, 2020, for the first time, the patient felt weakness in the left lower limb after several hours of driving. At that time, the CK level was 2,091 $\mu \mathrm{g} / \mathrm{l}$; except for slightly uneven shoulder blades, no muscular abnormalities were observed. The muscle volume was normal and no evidently abnormal distribution was evidenced by muscle MRI. However, 20 days later, the CK level was 11,262 $\mu \mathrm{g} / 1$ for no apparent reasons. Following hormone therapy, the CK level decreased to $1,179 \mu \mathrm{g} / \mathrm{l}$. In addition to increased CK, the patient also had increased total bilirubin $(61.6 \mu \mathrm{mol} / \mathrm{l})$ and direct bilirubin $(16.7 \mu \mathrm{mol} / \mathrm{l})$. Since disease onset, the patient's weight did not significantly vary and no family member showed similar symptoms. Except for the persistently elevated CK level, the patient did not show numbness, tingling, or pain in his hands or feet and other general physical symptoms were normal, including eating, urination, defecation and cranial nerve function.

The patient showed a normal cognitive function in the neuropsychological test via the Minor Mental State Examination (33). On physical examination, the patient presented approximately average muscle strength and volume. Muscle strength was graded according to the Medical Research Council scale (34): Neck flexors 5-/5, neck extensors 5/5, bilateral shoulder abductors 5-/5, left elbow flexors/extensors 5/5, right elbow flexors/extensors $5 / 5$, bilateral hip flexors/extensors, 


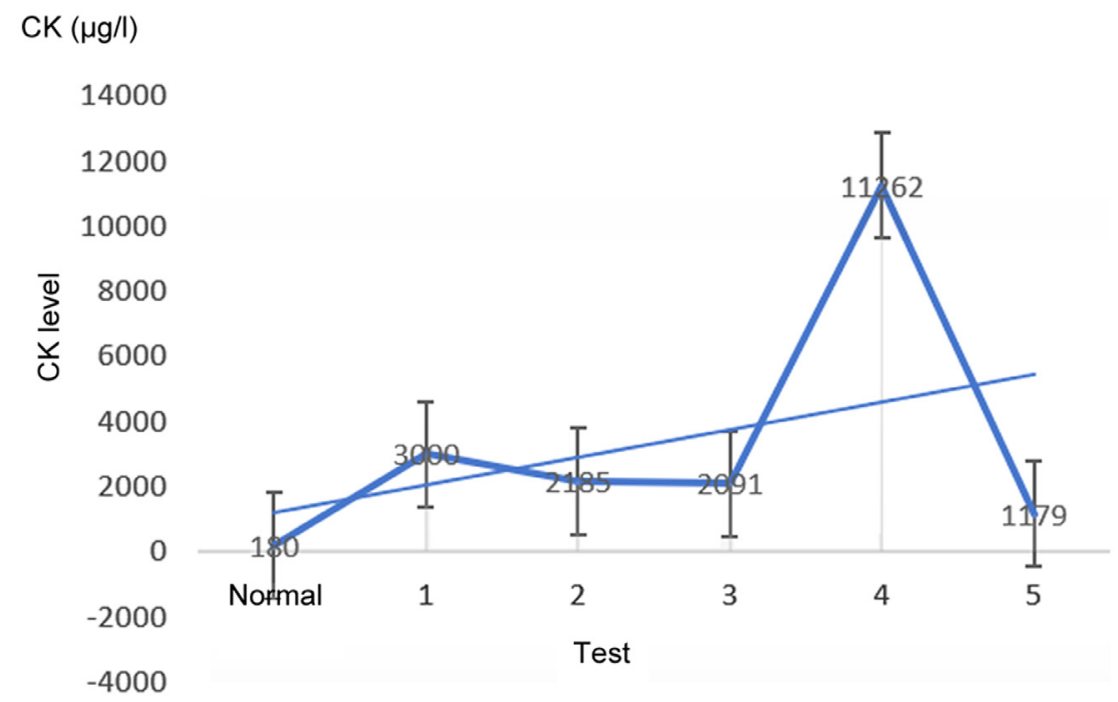

Figure 2. The five CK examinations. The horizontal axis represents the five CK examinations conducted since symptom onset, whereas the vertical axis represents the changes in CK levels $(\mu \mathrm{g} / \mathrm{l})$. The straight line represents the continuous increase in the CK level of the proband, whereas the fluctuating line represents the changes in the $\mathrm{CK}$ levels. $\mathrm{CK}$, creatine kinase.

bilateral wrist flexors/extensors, knee flexors/extensors and ankle dorsiflexors/plantar flexors 5-/5. No extraocular, facial, or bulbar muscle weakness or significant muscle atrophies were detected.

Laboratory examination showed a regular blood routine, serum potassium, erythrocyte sedimentation rate and C-reactive protein level. Markedly, the CK had been maintained at a high-level, reaching a maximum of 11,262 $\mu \mathrm{g} / \mathrm{l}$, which is 50-fold higher than the physiological level and independent of symptom severity (Fig. 2). The CK-MB and lactate dehydrogenase of the patient increased to 96 and $356 \mu \mathrm{g} / \mathrm{l}$, respectively, which may cause inflammatory myositis (35). Electromyography examination showed no noticeable myopathy changes, including motor unit action potentials with short duration, small amplitude and increased polyphasic potentials. Muscle MRI did not show abnormal signs of asymmetric muscle involvement. Muscle biopsy revealed a muscle tissue morphology consistent with normal muscle tissue and the results of immunohistochemical staining were normal. Genetic testing was performed after LGMD was diagnosed.

Genetic analysis. The average depth of sequencing was 257.15x; coupled with the results of Sanger sequencing verification, high sequencing reliability was obtained. WES data were filtered to exclude non-genetic variants and then compared with the dbSNP and $1000 \mathrm{G}$ databases. Subsequently, two likely pathogenic compound heterozygous variants in CAPN3 (NM_000070.2) (predicted by bioinformatics analyses) were validated by Sanger sequencing in the proband, a missense variant c.2120A>G/p.(Asp707Gly) (NM_000070.2: c.2120A>G, NP_000061.1: p.(Asp707Gly)) and deletion variant c.2201_2202delAT/p.(Tyr734*) [NM_000070.2: c.2201_2202delAT, NP_000061.1: p.(Tyr734*)], inherited from his father and mother, respectively.

The missense variant c.2120A>G/p.(Asp707Gly) in exon 20 resulted in a single-nucleotide polymorphism (A-G) at site 2120 in the coding region of CAPN3 (NM_000070.2), which introduced an aspartic acid residue that replaced a glycine in codon 707 (Fig. 3A). This variant was previously reported in multiple ( $>10)$ homozygous or compound heterozygous patients with LGMDs (PM3-PVS) (36), which co-segregated within a family (PP1-PM) (37). The normal population database includes this variant; its frequency is $0.0148 \%$ (42/282854, gnomAD; PM2); bioinformatics analysis software SIFT, PolyPhen2 and Mutation Taster consistently predicted the variant to be harmful (PP3). In addition, the variant is included in ClinVar as a 'pathogenic/suspected pathogenic variant' (36). According to available evidence, the variant is defined as pathogenic (PM3-PVS+PM2+PP1-PM+PP3) based on the 2015 ACMG guidelines for sequence variant interpretation (38).

A novel deletion variant c.2201_2202delAT/p.(Tyr734*) occurred in exon 21 of CAPN3 (NM_000070.2), which resulted in a variant at site 734 of the encoded protein that converted a tyrosine residue into a stop codon (Fig. 3B), which may cause protein truncation or activate nonsense-mediated CAPN3 mRNA degradation, thereby affecting the function of the protein product encoded by $C A P N 3$ (PVS1). This variant was not detected in the normal population database (PM2); in the trans-position, the pathogenic variant c.2201_2202delAT/p. (Tyr734*) was detected (PM3). According to the 2015 ACMG guidelines (38) for sequence variant interpretation, this variant is defined as pathogenic (PVS1+PM2+PM3).

The probability of these variants occurring in the population is extremely low and neither variant was not found in 200 identical ethnic healthy, unrelated controls from the Shandong population with the WT genotype. Based on the data of the present study and the characteristics of autosomal recessive inheritance, it was found that the CAPN3 compound heterozygous variants (c.2120A $>\mathrm{G}$ and c.2201_2202delAT) co-segregated with the LGMDR1 phenotypes in this threeperson family.

Multiple sequence alignment and molecular structure modeling. Multiple sequence alignment was performed to analyze protein sequence conservation. This analysis of CAPN3 protein from six species, including sheep, pig, cow, 


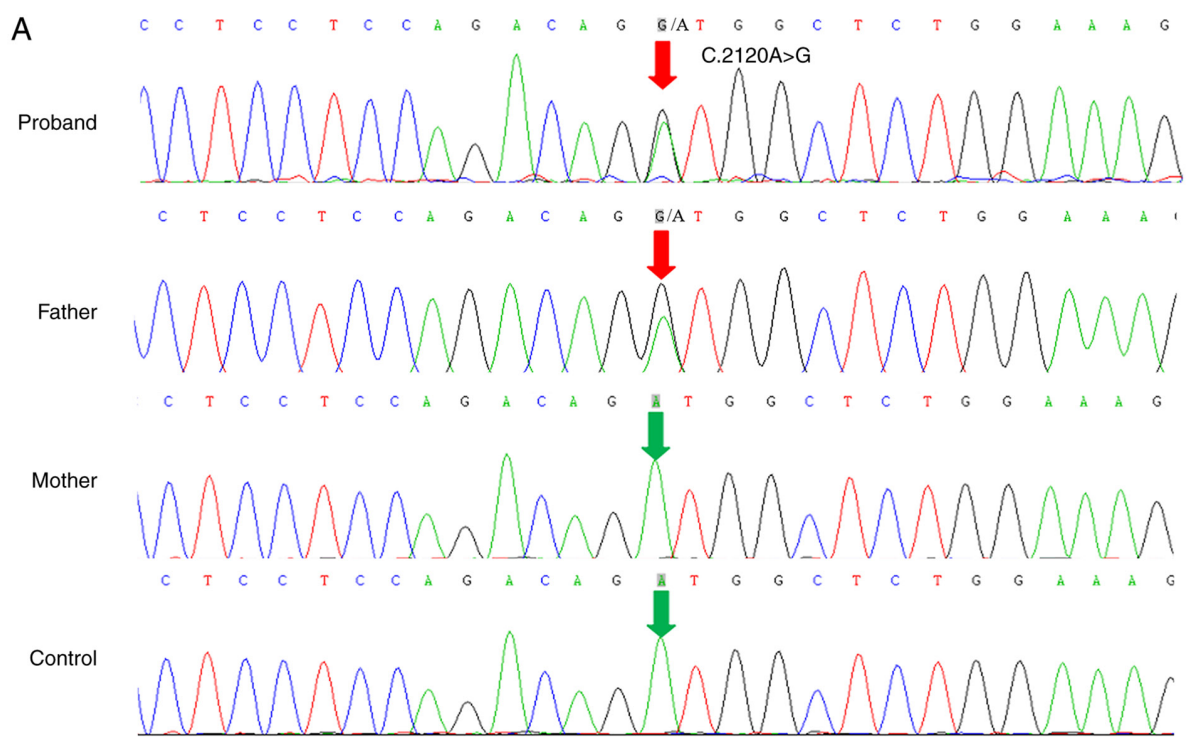

B

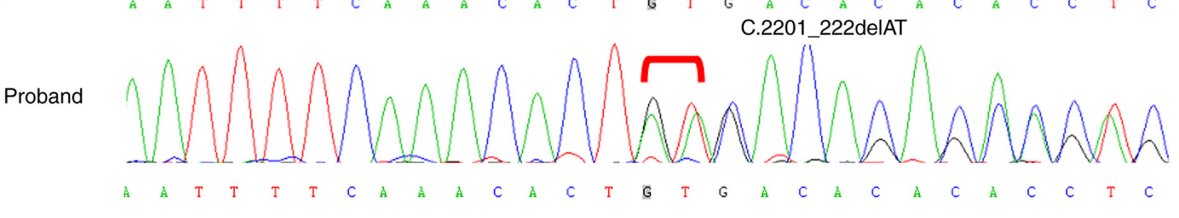

Mother

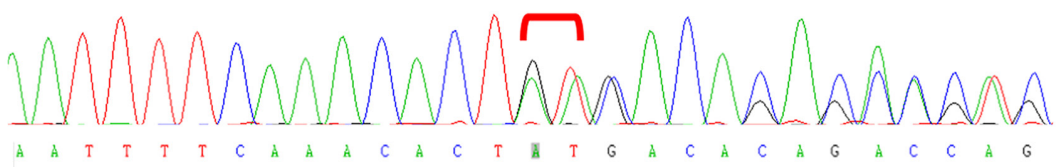

Father

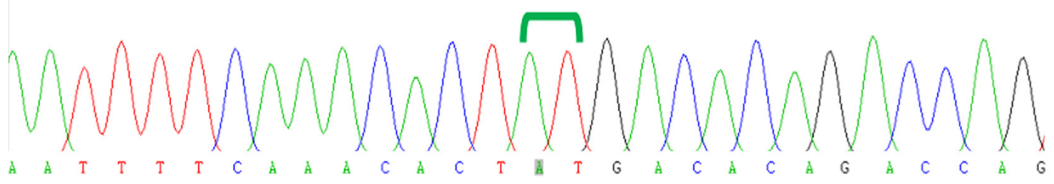

Control

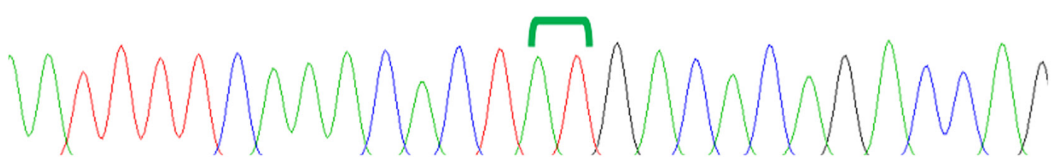

Figure 3. Sequence chromatograms of the variants in the family. (A) Sequence chromatograms of c.2120A>G/p.(Asp707Gly). The red arrow indicates the variant leads to an aspartic acid substitution for glycine at codon 707, which was inherited from his father. The normal sequence is shown below. (B) Sequence chromatograms of c.2201_2202delAT/p.(Tyr734*). The red line denotes that the variant leads to a premature termination codon at codon 734, which was inherited from the proband's mother, causing a variant from tyrosine to a stop codon. The normal sequence is shown below (CAPN3, NM_000070.2; NP_000061.1) p.(Asp707Gly).

p.Asp707

p.Tyr734

Homo sapiens

Chlorocebus sabaeus

Pan troglodytes

Rattus norvegicus

Bos taurus

Ovis aries

Sus scrofa

Consensus

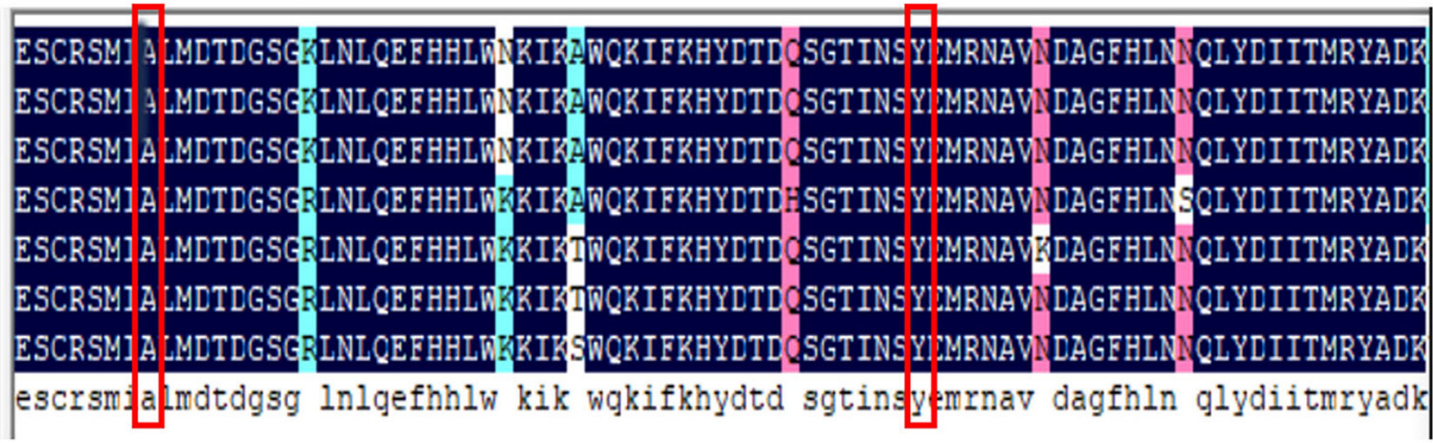

Figure 4. Alignment of multiple CAPN3 sequences. The regions of the two variants p.(Asp707Gly) and p.(Tyr734*) were highly conserved among different species, which are indicated by the red rectangles. 

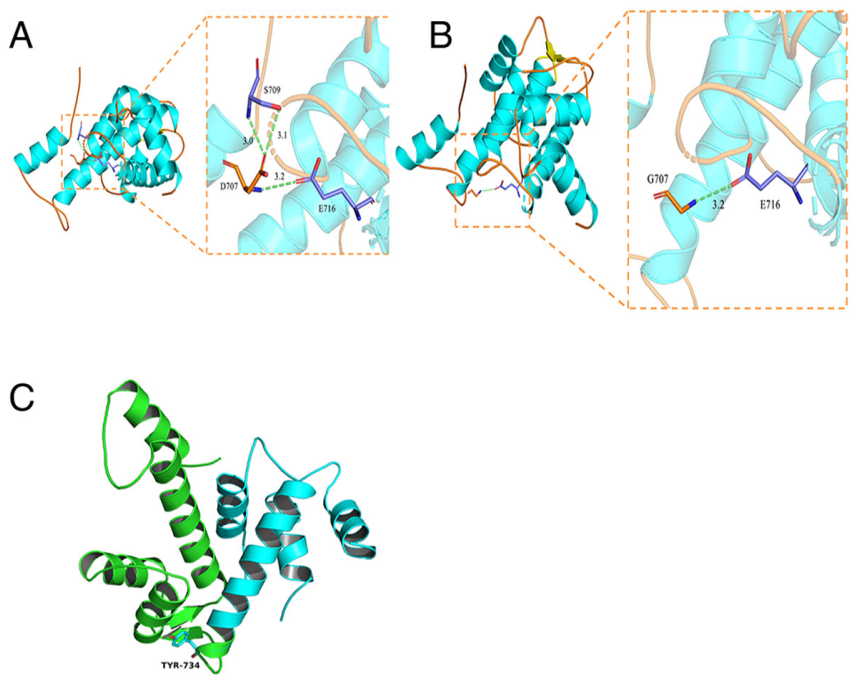

Figure 5. Three-dimensional structure model of CAPN3 protein. (A) Whole and local magnification of the PEF domain where wild-type Asp707 is localized. (B) Protein modeling shows the whole and local structure changes after the point variant localized at 707. (C) Model protein is in green and protein with the PEF domain variant in Tyr734 is in blue. PEF, penta-EF-hand.

rat, chimpanzee and monkey, showed that the aspartic acid and tyrosine residues at positions 707 and 734 of CAPN3 are highly conserved (Fig. 4).

A homologous PDB protein sequence was downloaded from the RCSB database (rcsb.org/) (PDB code 4OKH) and PyMOL-1 software was used to simulate the CAPN3 threedimensional protein structure (Fig. 5). Fig. 5A and B shows the interaction between residue 707 (p.Asp707) with surrounding amino acids before and after the variant. The aspartic acid at position 707 and serine at 709 formed two hydrogen bonds (indicated by green dotted lines, 3.0 and 3.1 represent the hydrogen bond distance between Asp707 and surrounding residues) and glutamic acid at site 716 forms a hydrogen bond (indicated by 3.2) with aspartic acid at position 707. Due to a point variant, the glycine at position 707 fails to form hydrogen bonds with surrounding amino acid residues. Compared with the WT sequence, the acid residue on position 707 lacks interactions with serine residues, weakening the interaction between the residue at position 707 with the surrounding amino acids, affecting overall protein stability. Fig. 5C shows that the deletion due to the tyrosine 734 nonsense variant resulted in a truncated protein and functional impairment.

\section{Discussion}

The present study observed a sporadic male case of LGMDR1 and identified two compound heterozygous variants in CAPN3, namely c.2120A>G/p. (Asp707Gly) and c.2201_2202delAT/p. (Tyr734*), which co-segregated with the LGMDR1 phenotypes in the proband's family. The genetic analysis of this family showed that the parents of this proband were obligate heterozygotes, as evidenced by Sanger sequencing and carried one CAPN3 pathogenic variant each. Therefore, it was considered that this proband had an autosomal recessive inheritance (AR-LGMDR1). Each sibling of this proband had a $25 \%$ possibility of being affected at conception, a $50 \%$ possibility of being an asymptomatic carrier and a $25 \%$ possibility of being unaffected but not a carrier. In silico analysis of these $C A P N 3$ variants revealed all deleterious results according to the ACMG guidelines for sequence variant interpretation. Additionally, the heterozygous compound variants were absent from 200 healthy, unrelated controls from the same ethnic group in the Shandong population with WT genotypes, suggesting they are pathogenic variants responsible for the LGMDR1 phenotypes.

Genetic analysis revealed a novel frameshift variant c.2201_2202delAT at the heterozygous state in exon 21 and a known missense variant (c.2120A $>$ G) in exon 20 of CAPN3 (36). Notably, both parents were carriers of pathogenic variants, as evidenced by Sanger sequencing, without related neuromuscular weakness or atrophies and normal CK levels. Therefore, it was considered that this proband possessed autosomal recessive inheritance. The CAPN3 missense variant p.(Asp707Gly) reportedly causes autosomal recessive inheritance, leading to severe and progressive clinical features and typical and evident muscular weakness and atrophies $(11,36,37,39)$. Missense $C A P N 3$ variants not only affect calpain 3 enzymatic activity, but also affect binding between protein molecules, thereby affecting protein integrity (20). Notably, previous studies showed that $C A P N 3$ variants can also cause the autosomal dominant pattern of LGMDD4 and deletion variants c.643_663del21, c.598_612del15, as well as missense variant c. $1333 \mathrm{G}>\mathrm{A}(8,40,41)$ can trigger similarly mild clinical features in the case of LGMDR1. Nevertheless, in the present study, the newly identified c.2201_2202delAT deletion variant contributed to the autosomal recessive LGMDR1 pattern because of genetic heterogeneity. Although this novel deletion created a truncated calpain 3 protein, the prognosis for LGMDR1 is unpredictable depending on whether the mutated site is located in a critically functional area (42).

It is well-known that missense variants in $C A P N 3$ are the most common type and frequently occur in the cysteine protease and PEF domain of calpain 3. The PEF domain binds four $\mathrm{Ca}^{2+}$ ions per protomer through $\mathrm{EF} 1, \mathrm{EF} 2, \mathrm{EF} 3$ and EF5 and three $\mathrm{Ca}^{2+}$-binding EF-hands are concentrated near the protease core, which may facilitate calpain 3 homodimerization and calcium ion binding and radically alter the local charge within the dimer during $\mathrm{Ca}^{2+}$ signaling $(20,32)$. The two $C A P N 3$ variants identified in the present study are respectively located in EF2 and EF3 of the PEF domain and may significantly change the 3D structure and disrupt the conformational stability of domain IV, thereby affecting the transmission of calcium signals.

Hyper-creatine kinase-emia refers to a clinically asymptomatic condition in which high serum CK levels are accidentally found during physical examination. CK levels may fluctuate, typically 5-80-fold above physiological levels. Among the reported cases, the serum $\mathrm{CK}$ level is elevated in almost all patients with hyper-creatine kinase-emia, showing values more than 10-fold higher than the normal level but has no apparent relationship with disease severity $(4,43)$. Fanin et al $(44)$ found that 6 of 58 patients with $C A P N 3$ variants presented with hyper-creatine kinase-emia and were asymptomatic, whereas only one was heterozygous for the c.550delA variant. A cohort study showed a frequency of $1.9 \%$ in German patients with 
LGMD who carried the deletion variant c.550delA in CAPN3, exhibiting sustained and isolated hyper-creatine kinaseemia (45). Notably, the calpainopathy-related hyper-creatine kinase-emia in the present study was diagnosed at the age of 18 years based on elevated $\mathrm{CK}$ levels but without myopathic symptoms. However, genetic analysis revealed $C A P N 3$ variants. Notably, few patients with hyper-creatine kinase-emia are asymptomatic or present only mild muscle weakness, indicating that very mild, late-onset calpainopathy phenotypes occurred in the proband in the present study.

Notably, some patients with normal CK levels present myopathy-related symptoms. Furthermore, the clinical phenotype of LGMDR1 is highly heterogeneous. The clinical phenotypes may completely differ, even between siblings with the same variant. In addition, the lack of a definitive correlation between gene variant sites and variant types $(23,46,47)$ further hinders accurate diagnoses. Thus, the severity and prognosis cannot be predicted based on the variant type and location alone (46). Severe cases may suffer disabilities during adolescence, which seriously affects motor abilities and eventually leads to the patient requiring a wheelchair; mild cases only present with mild myasthenia, hyper-creatine kinase-emia, or pseudometabolic myopathy in adulthood (42). Simple hypercreatine kinase-emia may be either a mild form of LGMDR1 or the preclinical stage of LGMDR1. The patient can be asymptomatic for many years but progressive weakness symptoms can appear over time. The high clinical heterogeneity of this disease is remarkable. Therefore, clinically asymptomatic hyper-creatine kinase-emia should be carefully investigated.

By performing muscular biopsies, Fanin et al (13) found that the pathological score of muscle tissue and degrees of fiber regeneration and degeneration in patients with LGMDR1 may be related to disease development. However, in the present case study, the male proband presented normal results. However, the pathogenesis of muscle changes caused by $C A P N 3$ variants requires further investigation.

In summary, the present study determined the genetic etiology of LGMDR1 in a family with $C A P N 3$ variants and performed genetic counseling for the proband, suggesting a genetic explanation for the simple hyper-creatine kinase-emia. The present study expanded the current clinical and genetic spectrum of LGMDR1, providing useful insights for further research on the pathogenesis of LGMDs and accelerating the development of prenatal diagnosis.

\section{Acknowledgments}

Not applicable.

\section{Funding}

The present study was supported by grants from the National Natural Science Foundation (NSFC; grant no. 81371499) and National Key Research and Development Program of China (grant nos. 2016YFC1000306 and 2005DKA32408).

\section{Availability of data and materials}

The datasets used and/or analyzed during the current study have been submitted to the ClinVar database [accession numbers VCV000468648.8 (https://www.ncbi.nlm.nih.gov/ clinvar/variation/468648/) and VCV000992898.1 (https:// www.ncbi.nlm.nih.gov/clinvar/variation/992898/)].

\section{Authors' contributions}

$\mathrm{CZ}$ and $\mathrm{LX}$ were responsible for data curation; $\mathrm{CZ}$ and $\mathrm{XZ}$ performed formal analysis; SL and FC were responsible for funding acquisition; DL and LX performed clinical investigation. SL and FC were responsible for project administration; CZ, LX, XZ, DL, SL and FC acquired materials; SL and FC supervised the present study. CZ and LX confirm the authenticity of all the raw data. $\mathrm{CZ}$ wrote the original draft; and $\mathrm{CZ}$, SL and FC wrote, reviewed and edited the manuscript. All authors read and approved the final manuscript.

\section{Ethics approval and consent to participate}

The present study was approved by the ethics committee of the Affiliated Hospital of Qingdao University (approval no. qdfy20203789) and was conducted according to the Declaration of Helsinki.

\section{Patient consent for publication}

Not applicable.

\section{Competing interests}

The authors declare that they have no competing interests.

\section{References}

1. Wicklund MP: The limb-girdle muscular dystrophies. Continuum (Minneap Minn) 25: 1599-1618, 2019.

2. Nallamilli BRR, Chakravorty S, Kesari A, Tanner A, Ankala A, Schneider T, da Silva C, Beadling R, Alexander JJ, Askree SH, et al: Genetic landscape and novel disease mechanisms from a large LGMD cohort of 4656 patients. Ann Clin Transl Neurol 5: 1574-1587, 2018.

3. Oliveira Santos M, Ninitas P and Conceição I: Severe limb-girdle muscular dystrophy $2 \mathrm{~A}$ in two young siblings from GuineaBissau associated with a novel null homozygous mutation in CAPN3 gene. Neuromuscul Disord 28: 1003-1005, 2018.

4. Bushby KM: The limb-girdle muscular dystrophies-multiple genes, multiple mechanisms. Hum Mol Genet 8: 1875-1882, 1999.

5. Angelini $\mathrm{C}$ and Fanin M: Calpainopathy. In: GeneReviews ${ }^{\circledR}$. Adam MP, Ardinger HH, Pagon RA and Wallace SE (eds). University of Washington, Seattle, WA, 2005.

6. Straub V, Murphy A, Udd B; LGMD workshop study group: 229th ENMC international workshop: Limb girdle muscular dystrophies - Nomenclature and reformed classification Naarden, the Netherlands, 17-19 March 2017. Neuromuscul Disord 28: 702-710, 2018.

7. Landires I, Núñez-Samudio V, Fernandez J, Sarria C, Villareal V, Córdoba F, Apráez-Ippolito G, Martínez S, Vidal OM, Vélez JI, et al: Calpainopathy: Description of a novel mutation and clinical presentation with early severe contractures. Genes (Basel) 11: E129, 2020.

8. Martinez-Thompson JM, Niu Z, Tracy JA, Moore SA, Swenson A, Wieben ED and Milone M: Autosomal dominant calpainopathy due to heterozygous CAPN3 C.643_663del21. Muscle Nerve 57: 679-683, 2018.

9. Richard I, Hogrel JY, Stockholm D, Payan CA, Fougerousse F, Eymard B, Mignard C, Lopez de Munain A, Fardeau M and Urtizberea JA; Calpainopathy Study Group: Natural history of LGMD2A for delineating outcome measures in clinical trials. Ann Clin Transl Neurol 3: 248-265, 2016. 
10. Richard I, Broux O, Allamand V, Fougerousse F, Chiannilkulchai N, Bourg N, Brenguier L, Devaud C, Pasturaud P, Roudaut C, et al: Mutations in the proteolytic enzyme calpain 3 cause limb-girdle muscular dystrophy type 2 A. Cell 81: 27-40, 1995.

11. Leiden Database: Available online: https://databases.lovd.nl/ shared/genes/CAPN3 (accessed on September 2, 2020).

12. Park HJ, Jang H, Lee JH, Shin HY, Cho SR, Park KD, Bang D, Lee MG, Kim SM, Lee JH, et al: Clinical and pathological heterogeneity of korean patients with CAPN3 mutations. Yonsei Med J 57: 173-179, 2016.

13. Fanin M, Nascimbeni AC and Angelini C: Gender difference in limb-girdle muscular dystrophy: A muscle fiber morphometric study in 101 patients. Clin Neuropathol 33: 179-185, 2014

14. Fanin M and Angelini C: Progress and challenges in diagnosis of dysferlinopathy. Muscle Nerve 54: 821-835, 2016.

15. Angelini C, Nardetto L, Borsato C, Padoan R, Fanin M, Nascimbeni AC and Tasca E: The clinical course of calpainopathy (LGMD2A) and dysferlinopathy (LGMD2B). Neurol Res 32: 41-46, 2010.

16. Duguez S, Bartoli M and Richard I: Calpain 3: A key regulator of the sarcomere? FEBS J 273: 3427-3436, 2006.

17. Pantoja-Melendez CA, Miranda-Duarte A, Roque-Ramirez B and Zenteno JC: Epidemiological and molecular characterization of a Mexican population isolate with high prevalence of limbgirdle muscular dystrophy type $2 \mathrm{~A}$ due to a novel calpain-3 mutation. PLoS One 12: e0170280, 2017.

18. Gallardo E, Saenz A and Illa I: Limb-girdle muscular dystrophy 2A. Handb Clin Neurol 101: 97-110, 2011

19. Ono Y, Ojima K, Shinkai-Ouchi F, Hata S and Sorimachi H: An eccentric calpain, CAPN3/p94/calpain-3. Biochimie 122 $169-187,2016$

20. Lasa-Elgarresta J, Mosqueira-Martín L, Naldaiz-Gastesi N, Sáenz A, López de Munain A and Vallejo-Illarramendi A: Calcium mechanisms in limb-girdle muscular dystrophy with CAPN3 mutations. Int J Mol Sci 20: E4548, 2019.

21. Hauerslev S, Sveen ML, Duno M, Angelini C, Vissing J and Krag TO: Calpain 3 is important for muscle regeneration: Evidence from patients with limb girdle muscular dystrophies BMC Musculoskelet Disord 13: 43, 2012.

22. Maki M: Structures and functions of penta-EF-hand calciumbinding proteins and their interacting partners: Enigmatic relationships between ALG-2 and calpain-7. Biosci Biotechnol Biochem 84: 651-660, 2020.

23. Sáenz A, Leturcq F, Cobo AM, Poza JJ, Ferrer X, Otaegui D, Camaño P, Urtasun M, Vílchez J, Gutiérrez-Rivas E, et al: LGMD2A: Genotype-phenotype correlations based on a large mutational survey on the calpain 3 gene. Brain 128: 732-742, 2005.

24. Toral-Ojeda I, Aldanondo G, Lasa-Elgarresta J, LasaFernández H, Fernández-Torrón R, López de Munain A and Vallejo-Illarramendi A: Calpain 3 deficiency affects SERCA expression and function in the skeletal muscle. Expert Rev Mol Med 18: e7, 2016.

25. Taveau M, Bourg N, Sillon G, Roudaut C, Bartoli M and Richard I: Calpain 3 is activated through autolysis within the active site and lyses sarcomeric and sarcolemmal components. Mol Cell Biol 23: 9127-9135, 2003.

26. Taghizadeh E, Rezaee M, Barreto GE and Sahebkar A: Prevalence, pathological mechanisms, and genetic basis of limb-girdle muscular dystrophies: A review. J Cell Physiol 234 7874-7884, 2019

27. Li H and Durbin R: Fast and accurate long-read alignment with Burrows-Wheeler transform. Bioinformatics 26: 589-595, 2010.

28. McKenna A, Hanna M, Banks E, Sivachenko A, Cibulskis K Kernytsky A, Garimella K, Altshuler D, Gabriel S, Daly M, et al: The Genome Analysis Tool kit: A MapReduce framework for analyzing next-generation DNA sequencing data. Genome Res 20: $1297-1303,2010$

29. Wang K, Li M and Hakonarson H: ANNOVAR: Functional annotation of genetic variants from high-throughput sequencing data. Nucleic Acids Res 38: e164, 2010.

30. Tran KT, Le VS, Bui HTP, Do DH, Ly HTT, Nguyen HT, Dao LTM, Nguyen TH, Vu DM, Ha LT, et al: Genetic landscape of autism spectrum disorder in Vietnamese children. Sci Rep 10 5034,2020

31. Schwede T, Kopp J, Guex N and Peitsch MC: SWISS-MODEL: An automated protein homology-modeling server. Nucleic Acids Res 31: 3381-3385, 2003.
32. Partha SK, Ravulapalli R, Allingham JS, Campbell RL and Davies PL: Crystal structure of calpain-3 penta-EF-hand (PEF) domain - a homodimerized PEF family member with calcium bound at the fifth EF-hand. FEBS J 281: 3138-3149, 2014

33. Arevalo-Rodriguez I, Smailagic N, Roqué I Figuls M, Ciapponi A, Sanchez-Perez E, Giannakou A, Pedraza OL, Bonfill Cosp X and Cullum S: Mini-Mental State Examination (MMSE) for the detection of Alzheimer's disease and other dementias in people with mild cognitive impairment (MCI). Cochrane Database Syst Rev 2015: CD010783, 2015.

34. Compston A: Aids to the investigation of peripheral nerve injuries. Medical Research Council: Nerve Injuries Research Committee. His Majesty's Stationery Office: 1942; pp. 48 (iii) and 74 figures and 7 diagrams; with aids to the examination of the peripheral nervous system. By Michael O'Brien for the Guarantors of Brain. Saunders Elsevier: 2010; pp. [8] 64 and 94 Figures. Brain 133: 2838-2844, 2010.

35. Rasmussen LH, Madsen HN and Ladefoged SD: Creatine phosphokinase MB and lactate dehydrogenase isoenzyme 1 in polymyositis. Scand J Rheumatol 14: 427-430, 1985.

36. Minami N, Nishino I, Kobayashi O, Ikezoe K, Goto Y and Nonaka I: Mutations of calpain 3 gene in patients with sporadic limb-girdle muscular dystrophy in Japan. J Neurol Sci 171: 31-37, 1999.

37. Park HJ, Jang H, Kim JH, Lee JH, Shin HY, Kim SM, Park KD, Yim SV, Lee JH and Choi YC: Discovery of pathogenic variants in a large Korean cohort of inherited muscular disorders. Clin Genet 91: 403-410, 2017.

38. Richards S, Aziz N, Bale S, Bick D, Das S, Gastier-Foster J, Grody WW, Hegde M, Lyon E, Spector E, et al; ACMG Laboratory Quality Assurance Committee: Standards and guidelines for the interpretation of sequence variants: A joint consensus recommendation of the American College of Medical Genetics and Genomics and the Association for Molecular Pathology. Genet Med 17: 405-424, 2015.

39. Ten Dam L, Frankhuizen WS, Linssen WHJP, Straathof CS, Niks EH, Faber K, Fock A, Kuks JB, Brusse E, de Coo R, et al: Autosomal recessive limb-girdle and Miyoshi muscular dystrophies in the Netherlands: The clinical and molecular spectrum of 244 patients. Clin Genet 96: 126-133, 2019.

40. Cerino M, Campana-Salort E, Salvi A, Cintas P, Renard D, Juntas Morales R, Tard C, Leturcq F, Stojkovic T, Bonello-Palot N, et al: Novel CAPN3 variant associated with an autosomal dominant calpainopathy. Neuropathol Appl Neurobiol 46: 564-578, 2020

41. Vissing J, Barresi R, Witting N, Van Ghelue M, Gammelgaard L, Bindoff LA, Straub V, Lochmüller H, Hudson J, Wahl CM, et al: A heterozygous 21-bp deletion in CAPN3 causes dominantly inherited limb girdle muscular dystrophy. Brain 139: 2154-2163, 2016.

42. Urtasun M, Sáenz A, Roudaut C, Poza JJ,Urtizberea JA, Cobo AM, Richard I, García Bragado F, Leturcq F, Kaplan JC, et al: Limbgirdle muscular dystrophy in Guipúzcoa (Basque Country, Spain). Brain 121: 1735-1747, 1998.

43. Fanin M and Angelini C: Protein and genetic diagnosis of limb girdle muscular dystrophy type 2A: The yield and the pitfalls. Muscle Nerve 52: 163-173, 2015.

44. Fanin M, Fulizio L, Nascimbeni AC, Spinazzi M, Piluso G, Ventriglia VM, Ruzza G, Siciliano G, Trevisan CP, Politano L, et al: Molecular diagnosis in LGMD2A: Mutation analysis or protein testing? Hum Mutat 24: 52-62, 2004

45. Hanisch F, Müller CR, Grimm D, Xue L, Traufeller K, Merkenschlager A, Zierz S and Deschauer M: Frequency of calpain-3 c.550delA mutation in limb girdle muscular dystrophy type 2 and isolated hyperCKemia in German patients. Clin Neuropathol 26: 157-163, 2007.

46. Fardeau M, Hillaire D, Mignard C, Feingold N, Feingold J, Mignard D, de Ubeda B, Collin H, Tome FM, Richard I, et al: Juvenile limb-girdle muscular dystrophy. Clinical, histopathological and genetic data from a small community living in the Reunion Island. Brain 119: 295-308, 1996.

47. Yu M, Zheng Y, Jin S, Gang Q, Wang Q, Yu P, Lv H, Zhang W, Yuan Y and Wang Z: Mutational spectrum of Chinese LGMD patients by targeted next-generation sequencing. PLoS One 12: $\mathrm{e} 0175343,2017$

This work is licensed under a Creative Commons

Attribution-NonCommercial-NoDerivatives 4.0 International (CC BY-NC-ND 4.0) License. 